\title{
Comprehensive characterization of a time- course transcriptional response induced by autotoxins in Panax ginseng using RNA-Seq
}

Bin Wu', Qiliang Long ${ }^{\dagger}$, Yuan Gao, Zi Wang, Tianwei Shao, Yanan Liu, Yong Li ${ }^{*}$ and Wanlong Ding*

\begin{abstract}
Background: As a valuable medicinal plant, the yield of Panax ginseng is seriously affected by autotoxicity, which is a common phenomenon due to continuous cropping. However, the mechanism of autotoxicity in $P$. ginseng is still unknown.
\end{abstract}

Results: In total, high throughput sequencing of 18 RNA-Seq libraries produced 996,000 000 100-nt reads that were assembled into 72,732 contigs. Compared with control, 3697 and 2828 genes were significantly up- and down-regulated across different tissues and time points, respectively. Gene Ontology enrichment analysis showed that 'enzyme inhibitor activity', 'carboxylesterase activity', 'pectinesterase activity', 'centrosome cycle and duplication' and 'mitotic spindle elongation' were enriched for the up-regulated genes. Transcription factors including AP2s/ERFs, MYBs, and WRKYs were up-regulated in roots after benzoic acid treatment. Moreover, reactive oxygen species, peroxidases and superoxide dismutase contigs were up-regulated in roots after benzoic acid treatment. Physiological and biochemical indexes showed that the proline and malondialdehyde content were restored to lower levels at a later stage after benzoic acid treatment. Benzoic acid inhibited the root hair development in a dose-dependent manner, and several differential expressed genes potentially involved in hair development were identified. Several key contigs in the flavonoid and ginsenoside biosynthesis pathways were repressed. Finally, 58,518 alternative splicing (AS) events from 12,950 genes were found after benzoic acid treatment. Interestingly, contigs in the ginsenoside biosynthetic pathway underwent AS, providing useful information about post-transcriptional regulation in $P$. ginseng.

Conclusions: This study revealed the stress-response molecular mechanisms in P. ginseng induced by benzoic acid.

Keywords: RNA-Seq, Panax ginseng, Autotoxicity, Benzoic acid

\section{Background}

Autotoxicity indicates that one plant releases allelochemicals into the adjacent environment, which directly or indirectly inhibit their own normal growth or that of their relatives in continuous cropping or short rotation [1-3]. Autotoxicity commonly occurs both in nature and cultivation and affects the yield and quality of plants [4-6]. The phenomenon was thought to be caused by soil-borne pathogens that gradually accumulated during continuous cropping at first. However, evidence showed that secondary micromolecular metabolites released by plants are

\footnotetext{
* Correspondence: liyong@implad.ac.cn; wlding@implad.ac.cn

${ }^{\dagger}$ Equal contributors

Institute of Medicinal Plant Development, Chinese Academy of Medical Sciences \& Peking Union Medical College, Beijing, China
}

important factors [2]. Autotoxicity has been found in many plants, such as rice [7], wheat [8], sweet potato [9], apple [10] and alfalfa [11]. Recently, medicinal plants were also reported to exhibit autotoxic phenomena [12-14]. Autotoxicity has been reported in more than 50 plant species thus far $[2,15]$.

Potential autotoxins have been explored for different compounds. For example, water-soluble organic acids, aliphatic aldehydes, lactones, long-chain fatty acids, naphthoquinones, anthraquinones, coumarins, tannins, steroids, alkaloids, cyanohydrins, sulfides, oil glycosides and purines have been isolated and identified as allelochemicals [2, 4]. Other types of autotoxins including phenolic compounds, flavones and terpenoids, and phenolic compounds are the main types of autotoxins 
$[2,4]$. The main phenolic acids are ferulic acid, o-hydroxy phenyl acetic acid, p-coumaric acid, ferulic acid, iso-ferulic acid, malic, critic, fumatic, and caffeic acids [16-18]. Microarrays have been used to investigate the gene expression profiling in plants in response to allelochemicals such as 2(3H)-benzoxazolinone [19], gallic acid [20], 3(3,4'-dihydroxyphenyl)-L-alanine [21], juglone [7] and ferulic acid [22]. However, DNA microarrays have limitations including background hybridization and known probe information. The recently developed next generation high throughput RNA sequencing (RNA-Seq) technique directly sequences transcripts to obtain a large dynamic range of expression levels and may provide comprehensive clues to solve this problem [23]. Furthermore, RNA-Seq can be used directly for transcriptome profiling for species with [24] or without [25] available genomes. Therefore, RNA-Seq has potential to replace microarray technology [26].

Panax ginseng is a highly valuable perennial herb with medicinal properties that is native to China and Korea [27] . However, continuous cropping of $P$. ginseng results in autotoxicity and a decline in biomass. To date, transcriptome studies of $P$. ginseng have focused on ginsenoside biosynthetic genes [28-31]. Transcriptome studies of $P$. ginseng after autotoxin treatment have not been reported. Benzoic acid is one of the major autotoxins identified in $P$. ginseng root exudates and rhizosphere soil. It significantly inhibited seed germination and growth $[32,33]$. Although the autotoxins in P. ginseng have been isolated and identified, their mechanisms remain unknown. In this study, we constructed RNA-Seq libraries using RNA extracted from roots, stems, and leaves treated with benzoic acid at six different time points and revealed enriched functional terms in response to this stress. Interestingly, several transcript factors were up-regulated in roots, suggesting the importance of transcription factors in response to benzoic acid. Moreover, peroxidase (POD) and superoxide dismutase (SOD) response to benzoic acid induction were identified. Several key contigs involved in the flavonoid and ginsenoside biosynthesis pathways were repressed. These results provide a comprehensive understanding of the $P$. ginseng response to benzoic acid stress and lay a foundation for improving the resistance or endurance of $P$. ginseng to autotoxins in the environment.

\section{Results}

RNA sequencing and de novo assembly

RNA samples were collected from roots, stems and leaves at 0 days post treatment (DPT), $1 \mathrm{DPT}, 3 \mathrm{DPT}, 5$ DPT, 7 DPT and 9 DPT after benzoic acid treatment. Then, RNA-Seq was performed to investigate DEGs or pathway responses to benzoic acid. In total, 18 RNA-Seq libraries generated approximately 996,000 000 clean reads of $100 \mathrm{nt}$ in length (Table 1). All the sequencing reads are deposited in the NCBI short read archive (SRA) under the accession number SRP049125. The reads were pooled together and assembled into reference sequences, yielding 72,732 contigs representing 272,053,772 total assembled bases for the $P$. ginseng transcriptome. The GC content was $38.55 \%$. The N50 and average length of assembled sequences were 1794 bp and 1428 bp, respectively (Additional file 1). This transcriptome assembly project has been deposited in DDBJ/EMBL/GenBank under the accession GDQW00000000.

\section{Annotation of the ginseng transcriptome}

The assembled transcripts were searched against the sequences in the NT database using the BLASTN algorithm (E-value $<10^{-6}$ ) for functional annotations. To obtain comprehensive annotation, coding regions were extracted using Trinity software [34]. These protein sequences were searched against the UniProt and NR databases using the BLASTP algorithm (E-value $<10^{-6}$ ). Complete annotation information was listed in Additional file 2. A total of $11,838,15,469$, and 21,807 genes were annotated in the UniProt, NR and NT databases, respectively. Finally, 28,139 genes were annotated, and the NT database had the largest match, followed by the NR and UniProt databases. BLAST was also performed against the KEGG database to annotate the metabolic pathways for each gene. A total of 2783 genes in 262 pathways were identified according to the KEGG database (Additional file 3).

\section{Calculation of gene expression}

Gene expression was measured by mapping RNA-Seq reads from 18 libraries to the assembled sequences. On average, $85 \%$ of total reads were successfully mapped to the assembled transcript sequences using Bowtie2 2.2.3 [35]. Subsequently, FPKM was adopted to quantify the expression of 72,732 contigs. Both pairs of PE reads with unique location were retained to calculate FPKM value in the following analysis to detect the DEGs associated with benzoic acid stress. There were 704,917,418 (71\% of the total) reads that were mapped back in pairs with unique locations on the assembled genes and used for the downstream FPKM calculation. The average FPKM value for all samples examined was 26. Additional file 4 shows the distributions of FPKM values followed by $\log 2$ transformation. In total, 26,973 genes with the minimum expression threshold of FPKM $>3$ were identified in at least one RNA-Seq library.

\section{Identification of DEGs and dynamic profile}

Each DEG was evaluated by the MA-plot-based method with random sampling model [36] to calculate the $P$ value by the pair-wise comparison of control samples 
Table 1 Summary of Illumina sequencing and transcriptome assemblies for RNA-Seq libraries

\begin{tabular}{|c|c|c|c|c|c|c|c|c|}
\hline Indcued time & Tissues & Reads & Length & Total bases & Mapping reads & Unique reads & Unigenes & SRA experiment IDs \\
\hline \multirow[t]{3}{*}{0 day } & root & $54,802,994$ & 100,100 & $5,480,299,400$ & $46,616,140$ & $38,539,562$ & 19,524 & SRX758350 \\
\hline & stem & $62,654,934$ & 100,100 & $6,265,493,400$ & $53,955,686$ & $44,640,828$ & 19,207 & SRX758356 \\
\hline & leaf & $62,304,990$ & 100,100 & $6,230,499,000$ & $53,976,979$ & $45,581,048$ & 18,886 & SRX758362 \\
\hline \multirow[t]{3}{*}{1 day } & root & $53,367,552$ & 100,100 & $5,336,755,200$ & $44,893,957$ & $36,983,148$ & 19,948 & SRX758351 \\
\hline & stem & $49,148,806$ & 100,100 & $4,914,880,600$ & $42,382,932$ & $35,029,638$ & 19,317 & SRX758357 \\
\hline & leaf & $63,531,810$ & 100,100 & $6,353,181,000$ & $54,699,654$ & $45,625,544$ & 19,328 & SRX758363 \\
\hline \multirow[t]{3}{*}{3 day } & root & $54,362,660$ & 100,100 & $5,436,266,000$ & $45,401,558$ & $37,587,578$ & 21,180 & SRX758352 \\
\hline & stem & $48,114,866$ & 100,100 & $4,811,486,600$ & $41,208,427$ & $33,669,930$ & 19,900 & SRX758358 \\
\hline & leaf & $55,411,400$ & 100,100 & $5,541,140,000$ & $47,776,074$ & $39,877,716$ & 20,088 & SRX758364 \\
\hline \multirow[t]{3}{*}{5 day } & root & $55,644,310$ & 100,100 & $5,564,431,000$ & $46,703,315$ & $38,601,890$ & 20,389 & SRX758353 \\
\hline & stem & $60,981,712$ & 100,100 & $6,098,171,200$ & $52,263,645$ & $43,831,390$ & 21,019 & SRX758359 \\
\hline & leaf & $54,073,298$ & 100,100 & $5,407,329,800$ & $45,762,381$ & $37,334,730$ & 19,633 & SRX758365 \\
\hline \multirow[t]{3}{*}{7 day } & root & $49,978,570$ & 100,100 & $4,997,857,000$ & $41,697,612$ & $34,220,122$ & 20,354 & SRX758354 \\
\hline & stem & $52,655,072$ & 100,100 & $5,265,507,200$ & $45,398,187$ & $37,654,496$ & 18,971 & SRX758360 \\
\hline & leaf & $56,194,980$ & 100,100 & $5,619,498,000$ & $48,170,802$ & $40,207,756$ & 18,385 & SRX758366 \\
\hline \multirow[t]{3}{*}{9 day } & root & $55,412,038$ & 100,100 & $5,541,203,800$ & $46,873,595$ & $38,947,342$ & 19,271 & SRX758355 \\
\hline & stem & $53,283,108$ & 100,100 & $5,328,310,800$ & $46,033,892$ & $37,871,864$ & 17,644 & SRX758361 \\
\hline & leaf & $54,119,488$ & 100,100 & $5,411,948,800$ & $46,471,434$ & $38,712,836$ & 18,776 & SRX758367 \\
\hline
\end{tabular}

and benzoic acid induced samples. The stringent cutoffs for DEGs included a three-fold or higher change and $P<0.001$. A total of 3697 and 2828 genes were identified with significantly up- and down-regulated expression, respectively, compared with control (Table 2). All the differential expressed genes could be found at Additional file 5. To validate the RNA-Seq results, qRT-PCR was

Table 2 The number of up- and down-regulated DEGs based on pair-wise comparison with 0 day control (fold change $>3$; $P<0.001$ )

\begin{tabular}{llll}
\hline Indcued time & Tissue & Up-regulated & Down-regulated \\
\hline 1 day & root & 104 & 39 \\
& stem & 63 & 41 \\
& leaf & 139 & 69 \\
3 day & root & 589 & 41 \\
& stem & 178 & 230 \\
& leaf & 68 & 72 \\
5 day & root & 328 & 55 \\
& stem & 1443 & 751 \\
& leaf & 536 & 264 \\
7 day & root & 204 & 61 \\
& stem & 189 & 663 \\
& leaf & 310 & 242 \\
9 day & root & 785 & 212 \\
& stem & 490 & 1698 \\
& leaf & 444 & 634 \\
\hline
\end{tabular}

carried out on 28 randomly chosen DEGs at 0 DPT, 1 DPT, 3 DPT, 5 DPT, 7 DPT and 9 DPT. The expression profiles based on RNA-Seq could be found at Additional file 6 . The qRT-PCR results were highly consistent with those from the RNA-Seq (Fig. 1). The Pearson correlation coefficient was 0.90, indicating that the qRT-PCR results were positively linearly related with the RNA-Seq results (Fig. 1). The RNA-Seq profiles of 28 randomly chosen DEGs and all the genes that described in the result parts were validated by qRT-PCR (Additional files 7, 8, 9, 10, 11 and 12). Genes with similar expression patterns might be functionally correlated [37]. In order to reveal the expression change after benzoic acid treatment, six time points and three tissues across 18 RNA-Seq libraries were used to identify statistically significant expression profiles and the genes associated with the profiles. To investigate the dynamic patterns of these DEGs after benzoic acid treatment, gene expression cluster analysis was performed using the STEM algorithms [38]. In brief, the STEM algorithm first selects a set of representative model profiles. Then the correlation coefficient for each gene was used to identify the most closely model profile. Then the profiles with statistically significant higher number of genes were selected using permutation test. STEM algorithm analyses of the expressed genes generated three significant clusters $(P<0.001)$ based on the expression profile similarity. Cluster 1 included 263 genes which increased sharply at early time points (3 DPT) after benzoic acid treatment in roots (Fig. 2). Genes in this cluster were predominantly 


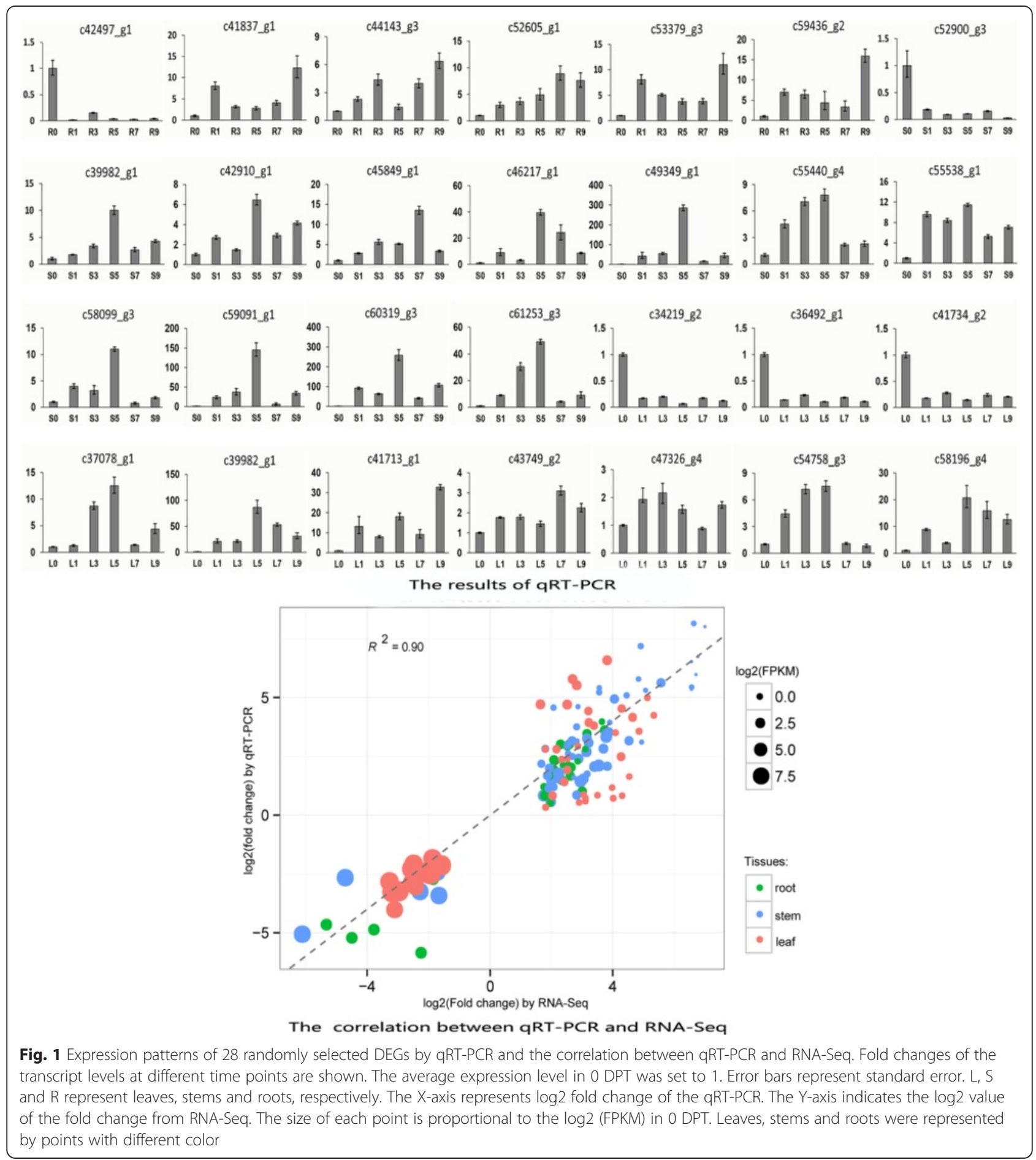

enriched in response to toxin metabolic process (3.4398E-4) and response to stress (3.9015E-3) based on GO analysis. However, for cluster 2 in the stems and cluster 3 in the leaves, the maximum abundance shifted to later time points (5 DPT) (Fig. 2). The gene numbers for these two clusters were 304 and 168, respectively. GO terms enriched in cluster 3 also included response to stress (7.0496E-4) and response to stimulus (1.3493E-3), suggesting the importance of the genes that response to stress after benzoic acid treatment. Within contrast to these two clusters, genes in cluster 2 were enriched in nuclease activity (3.4498E-6) and DNA metabolic process (8.3423E-5), respectively. 


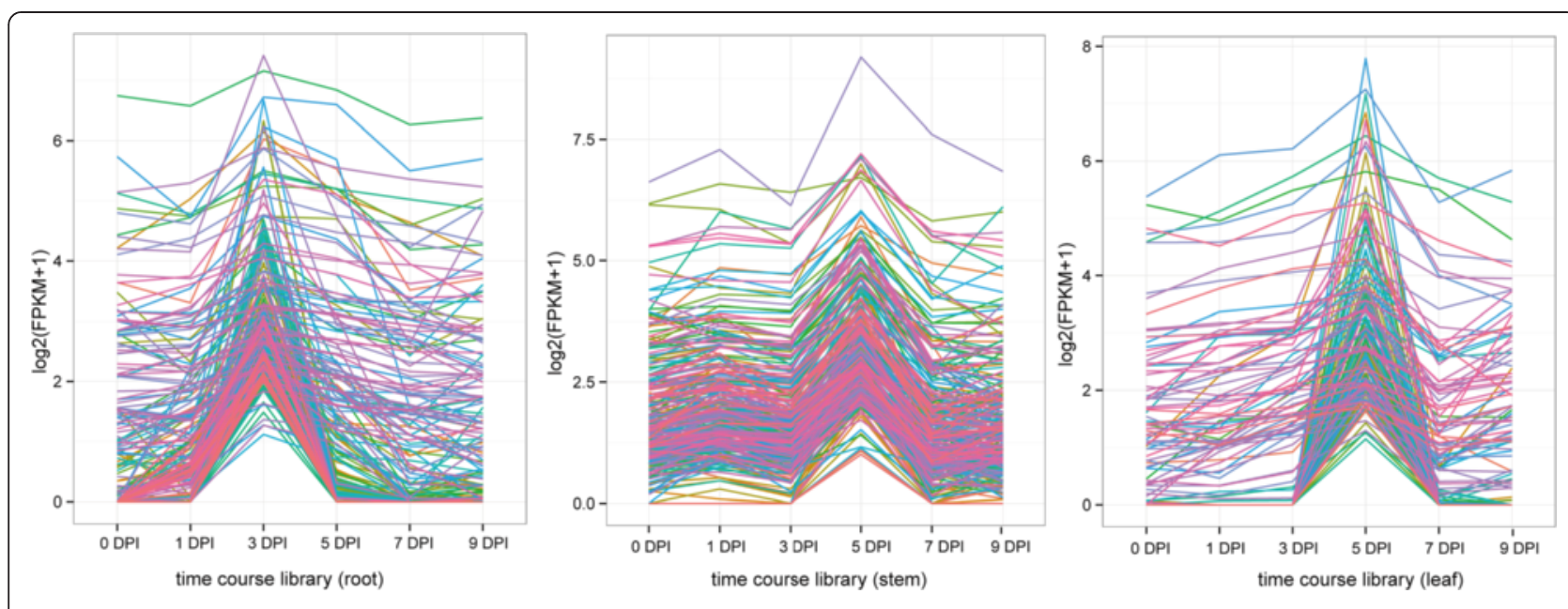

Fig. 2 Representative time-course profile clusters. Representative profile clusters for roots, stems and leaves. For each time point and each gene, the $\log 2(F P K M+1)$ is shown for the mean expression profile for each cluster

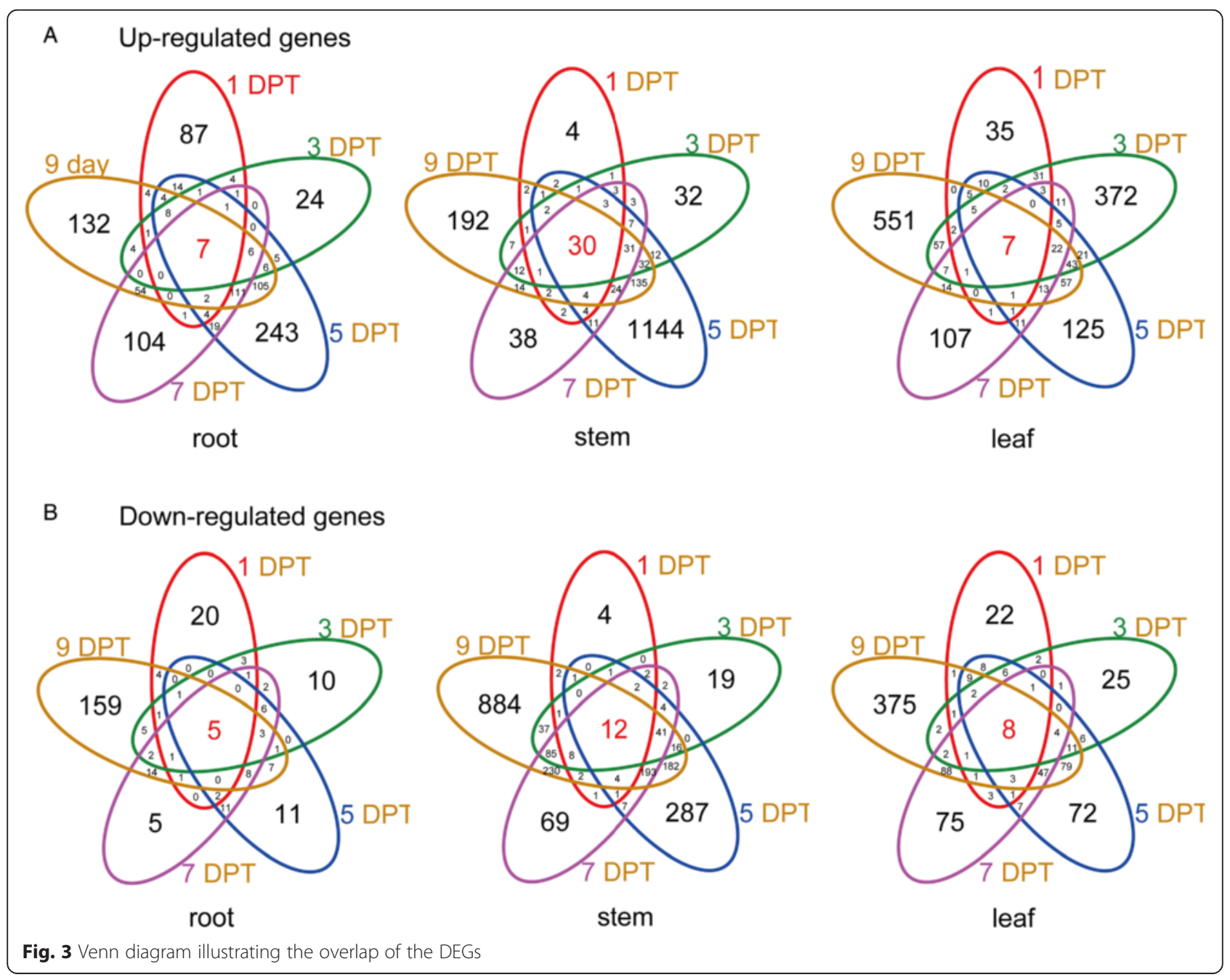




\section{DEGs overlap at six different time points}

DEGs that overlapped at all time points were genes induced by benzoic acid across the entire stress process. In total, seven contigs were induced across all time points (1 DPT, $3 \mathrm{DPT}, 5 \mathrm{DPT}, 7 \mathrm{DPT}$ and $9 \mathrm{DPT}$ ) in roots (Fig. 3). Among these, the EG45-like domain containing protein (c28773_g1) was significantly up-regulated in roots. The qRT-PCR validation for the gene was consistent with the result of RNA-Seq (Additional file 7).

In this study, 30 contigs including five transcription factors were up-regulated across all time points in stems (Fig. 3). The function of these transcription factors was deduced from the homology protein of UniProt database in other species. Two MYB transcription factors (c49349_g1 and c59091_g1) can respond to ethylene, jasmonic acid and abscisic acid. Two No Apical Meristem (NAM) transcription factors (c53886_g5 and c48053_g1) functioned in multidimensional cell growth and the negative regulation of the abscisic acid activated signaling pathway. Transcription factor WRKY (c46217_g1) played a critical role in the response to chitin. The expression profiles based RNA-Seq and qRT-PCR validation for the above-mentioned transcript factors could be found at Additional file 7 .

In the leaves, seven genes were up-regulated across all the time points (Fig. 3). The plant invertase/pectin methylesterase inhibitor has been reported as a stress-response gene [39]. In this study, we also found that one plant invertase/pectin methylesterase inhibitor (c41713_g1) gene was up-regulated in the leaves (Additional file 7).

We also observed down-regulated genes across all the time points. For example, one unigene that coded for zinc-binding dehydrogenase (c8930_g1) was downregulated in roots, and another unigene that coded for calcium-binding protein with EF-hand domain (c41722_g1) was down-regulated in stems (Additional file 7 ). These results are consistent with previous reports that the lipophilicity of benzoic and cinnamic acids could impact the ion uptake and root elongation in cucumber [40]. All the differentially expressed genes at all time points could be found at Additional file 13 .

\section{GO functional enrichment analyses}

The function of the 3697 up-regulated DEGs was determined by GO enrichment analysis. The results showed that the up-regulated genes were enriched for 'transcription factor activity', 'carboxylesterase activity', and 'pectinesterase activity' (Fig. 4). Up-regulated genes were also involved in 'centrosome cycle and duplication' and 'mitotic spindle elongation'. Other enriched terms included 'response to water deprivation' $(P=3.6799 \mathrm{E}-3)$, consistent with previous findings that exposure of plant roots to ferulic acid can affect water usage [41]. We also found that 'polysaccharide metabolic process' $(P=4.5829 \mathrm{E}-4)$ and 'lignin metabolic process' $(P=2.5213 \mathrm{E}-9)$ were enriched.
Among the down-regulated genes, GO terms associated with 'cell wall organization or biogenesis', 'stress response', 'secondary metabolic processes' and 'ion binding' were enriched (Fig. 4). Down-regulated genes involved in 'polysaccharide metabolic process' (34 genes) and 'lignin metabolic process' (19 genes) showed significant responses to benzoic acid. Autotoxin usually led to a 20-50\% decrease in growth rate [2]. The photosystem is an important factor that influences plant growth. Contigs involved in 'photosystem' $(P=3.8496 \mathrm{E}-4)$ were significantly repressed, which can partly explain the reduction of production due to autotoxicity.

\section{Reactive oxygen species}

Reactive oxygen species (ROS) produced from various metabolic pathways can cause oxidative damage [42]. For example, ferulic acid can produce oxidative stress in rice [22]. Plants can produce reactive oxygen species (ROS) during abiotic stress as byproducts of aerobic metabolism. At the same time plant must have the ability to remove the toxin. Benzoic acid treatment can be regarded as abiotic stress, thus the investigation of the generation and removal of ROS can help us to understand the possible function of ROS upon benzoic acid treatment.

In this study, 35 ROS-related genes were annotated as 'response to reactive oxygen species' (GO: 0000302 and GO: 0072593). Among them, three contigs (c14304_g1, c42911_g1 and c34265_g1) were significantly up-regulated, suggesting that these genes might take part in the oxidative damage response upon benzoic acid induction. In leaves, c14304_g1 was up-regulated at an early stage (1 DPT). However, c42911_g1 and c34265_g1 were up-regulated at a later stage in roots (9 DPT). The RNA-Seq profiles and qRT-PCR validations for the above three ROS-related genes could be found at Additional file 8 .

We also investigate the differential expression levels of contigs coding POD and SOD. In total, we identified 64 contigs with peroxidase activity. Two PODs (c50418_g2 and c52462_g1) were up-regulated at 9 DPT in roots. We also identified nine contigs with SOD activity. Among them, c41673_g1 was up-regulated in roots (3 DPT) and leaves (5 DPT). The RNA-Seq profiles and qRT-PCR validations for two PODs genes (c50418_g2 and c52462_g1) and one SOD genes (c41673_g1) could be found at Additional file 8.

\section{Measurement of physiological and biochemical indexes of proline and malondialdehyde}

The impacts of benzoic acid over a concentration gradient of $2.5-250 \mathrm{mg} \cdot \mathrm{L}^{-1}$ on the accumulation of proline and malondialdehyde (MDA) was investigated. MDA is the product of lipid membrane peroxidation and is a marker of stress-induced damage [43]. In general, the content of 


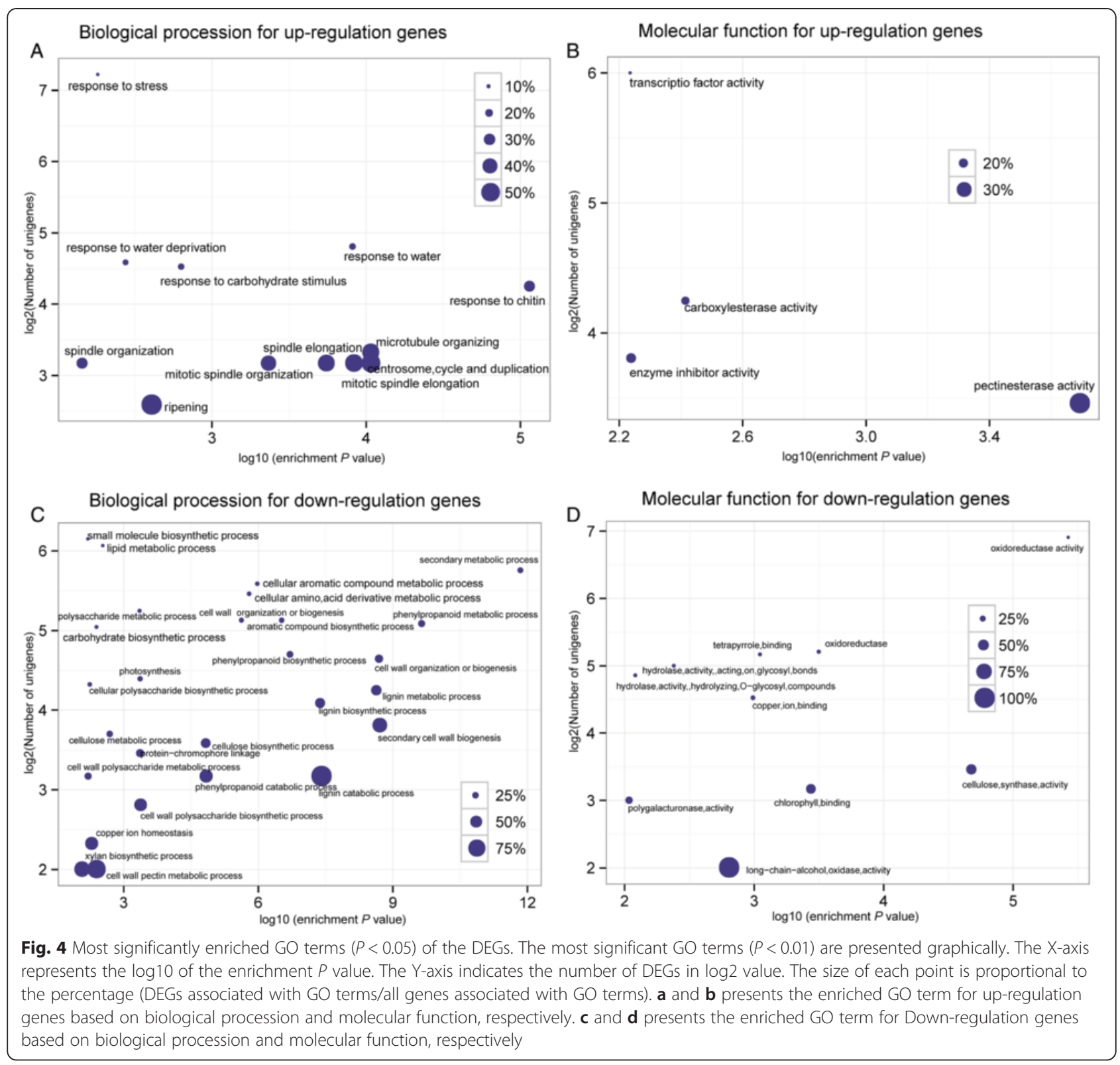

both free proline and MDA increased as the concentration of benzoic acid increased compared with control (Fig. 5). The accumulation of proline at 5 DPT was higher than at 0, 1, 3, 7 and 9 DPT. In the proline biosynthetic pathway, pyrroline-5-carboxylate synthetase (P5CS) and pyrroline5-carboxylate reductase (P5CR) were two important enzymes with important roles in turning glutamic acid into proline $[44,45]$. The expression level of P5CS (c61458_g1) showed a similar profile as proline. However, the expression level of P5CR (c61356_g1) was unchanged during benzoic acid treatment. For MDA (an index of lipid peroxidation), the content at 7 DPT was higher than at $0,1,3,5$ and $9 \mathrm{DPT}$, suggesting that benzoic acid treatment promoted lipid peroxidation at later stage. Ascorbate is catalyzed and converted into MDA by ascorbate peroxidase (APX) [46]. According to the RNA-Seq results, the expression of APX (c49700_g1) increased at the early stage and decreased at the later stage, consistent with the change in MDA content. The RNA-Seq profiles for P5CS, P5CR and APX, together with the qRT-PCR validations were shown at Additional file 9.

\section{Effect of autotoxicity on root hair development}

Autotoxins produced by plants inhibit their own growth directly or indirectly. For example, ferulic acid inhibited root elongation in rice [4]. Therefore, we also observed the root morphology after benzoic acid treatment. The 


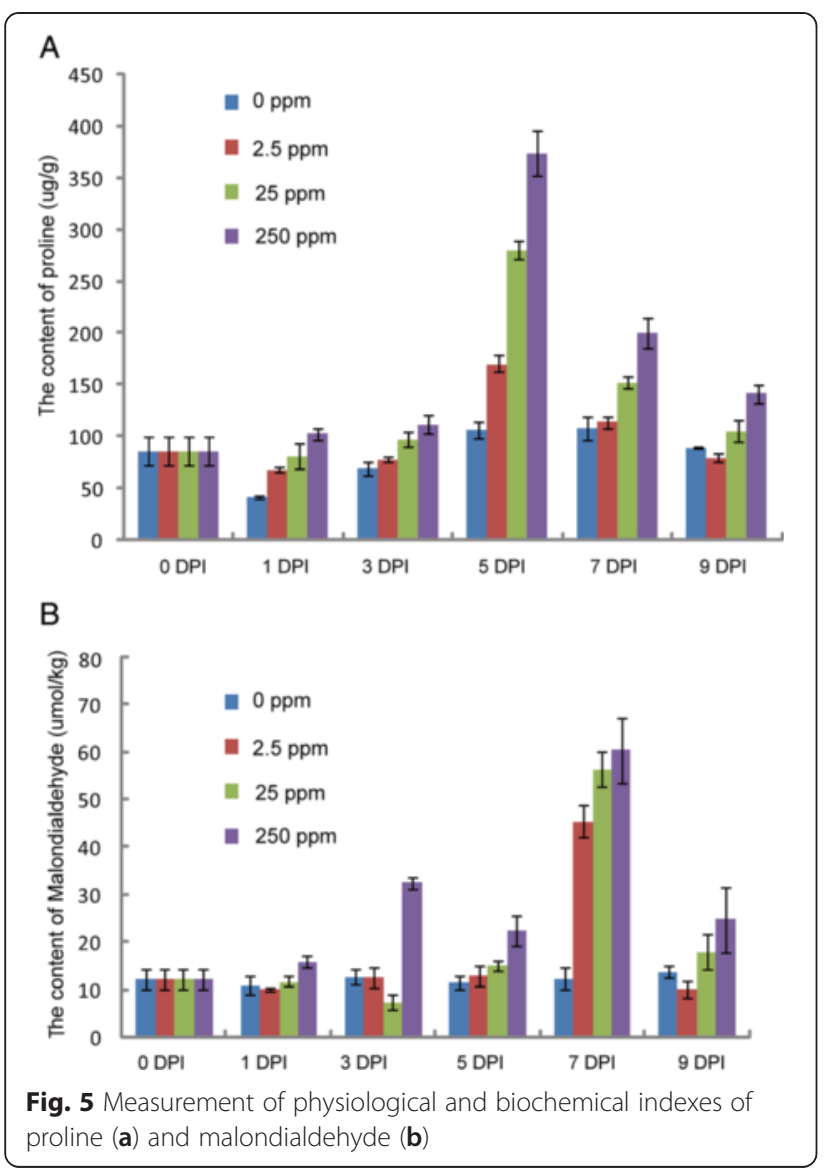

results showed that benzoic acid caused permanent morphological reductions in root hair density by observation of root morphology using stereomicroscope 4 . The adverse effect of autotoxins on root development increased as the concentration of benzoic acid increased (Additional file 14). The root hair growth was almost completely inhibited when the concentration of benzoic acid reached $250 \mathrm{mg} \cdot \mathrm{L}^{-1}$, suggesting that benzoic acid repressed the root hair in a concentration-dependent manner. The possible mechanism of the effect of benzoic acid on root hair formation was investigated. In total, 41 root hair related contigs were identified from the homology protein of UniProt database in other species, and the contigs (c60670_g1, c59443_g4, c47561_g2, c44448_g2 and c43137_g2) were highly expressed in roots compared with leaves and stems (Fig. 6). Among these root hair related contigs, four up-regulated (c35599_g1, c44448_g2, c47561_g2 and c54588_g1) and two down-regulated (c52527_g1 and c53982_g1) contigs might contribute to the low density of root hairs upon benzoic acid treatment. c35599_g1 was up-regulated at 3 DPT in roots; both c44448_g2 and c47561_g2 were up-regulated in stems (7 DPT and 9 DPT) and c54588_g1 was up-regulated in roots (9 DPT). Two down-regulated contigs were observed in stems. The qRT-PCR validations with RNA-Seq profiles for the above nine genes could be found at Additional file 10.

\section{Flavonoid and ginsenoside biosynthetic pathways upon benzoic acid treatment}

Previous studies revealed that flavonoids were also involved in autotoxicity and resulted in root growth inhibition and cell division reduction in root meristematic regions [47]. Chalcone synthase (CHS), chalcone isomerase $(\mathrm{CHI})$, flavanone 3-hydroxylase $(\mathrm{F} 3 \mathrm{H})$, flavonoid 3' 5'-hydroxylase (F3'5'H), flavonoid 3'-hydroxylase (F3'H), anthocyanidin synthase (ANS), and UDPglucose:anthocyanidin 3-O-glucosyltransferase (A3GT) genes are key enzymes in the flavonoid biosynthesis pathway $[48,49]$. In this study, we identified one CHS unigene (c57094_g1), two CHI contigs (c366_g1 and c45255_g1), one F3H contigs (c42137_g1) and two A3GT contigs (c43015_g1 and c63923_g1). Interestingly, CHI (c45255_g1) was repressed in stems at the later stage (5 DPT and 7 DPT) after benzoic acid treatment. F3H (c42137_g1) was also repressed in roots at the later stage (7 DPT and 9 DPT). The RNA-Seq profiles and qRT-PCR validations for $\mathrm{CHI}$ and $\mathrm{F} 3 \mathrm{H}$ could be found at Additional file 11.

Ginsenosides are the major bioactive components of $P$. ginseng. Due to their important pharmacological effects, the ginsenoside biosynthetic pathway has been widely investigated [28-31]. Cytochrome P450 involved in the ginsenoside biosynthetic pathway might represent the largest family and catalyze most of the oxidation steps during the biogenesis of plant secondary metabolism. In this study, 20 potential cytochrome P450 contigs were down-regulated and 13 were up-regulated. The remaining contigs involved in the ginsenoside biosynthetic pathway were down-regulated upon benzoic acid treatment. The down-regulated DEGs included three farnesyl diphosphates (FPS), two-geranylgeranyl pyrophosphate synthase (GPS), one hydroxymethylglutaryl-CoA synthase (HMGS) and two UGT (UDP-glycosyltransferases). The RNA-Seq profiles and qRT-PCR validations for the two FPS (c47877_g1, c48923_g5,), two GPS (c47128_g1, c7968_g1), one HMGS (c60578_g1) and two UGT (c51692_g1, c64659_g1) for CHI and F3H could be found at Additional file 11.

\section{The expression of transcription factors upon benzoic acid} treatment

TFs function in the abiotic stress tolerance response, and overexpression of these TFs generated transgenic plants with significant tolerance to abiotic stresses [50-53]. A total of 64 TFs were significantly up-regulated by benzoic acid, and TF expression levels were higher in roots than in other tissues (Fig. 7). In total, $10 \%$ of TFs in the TF family 


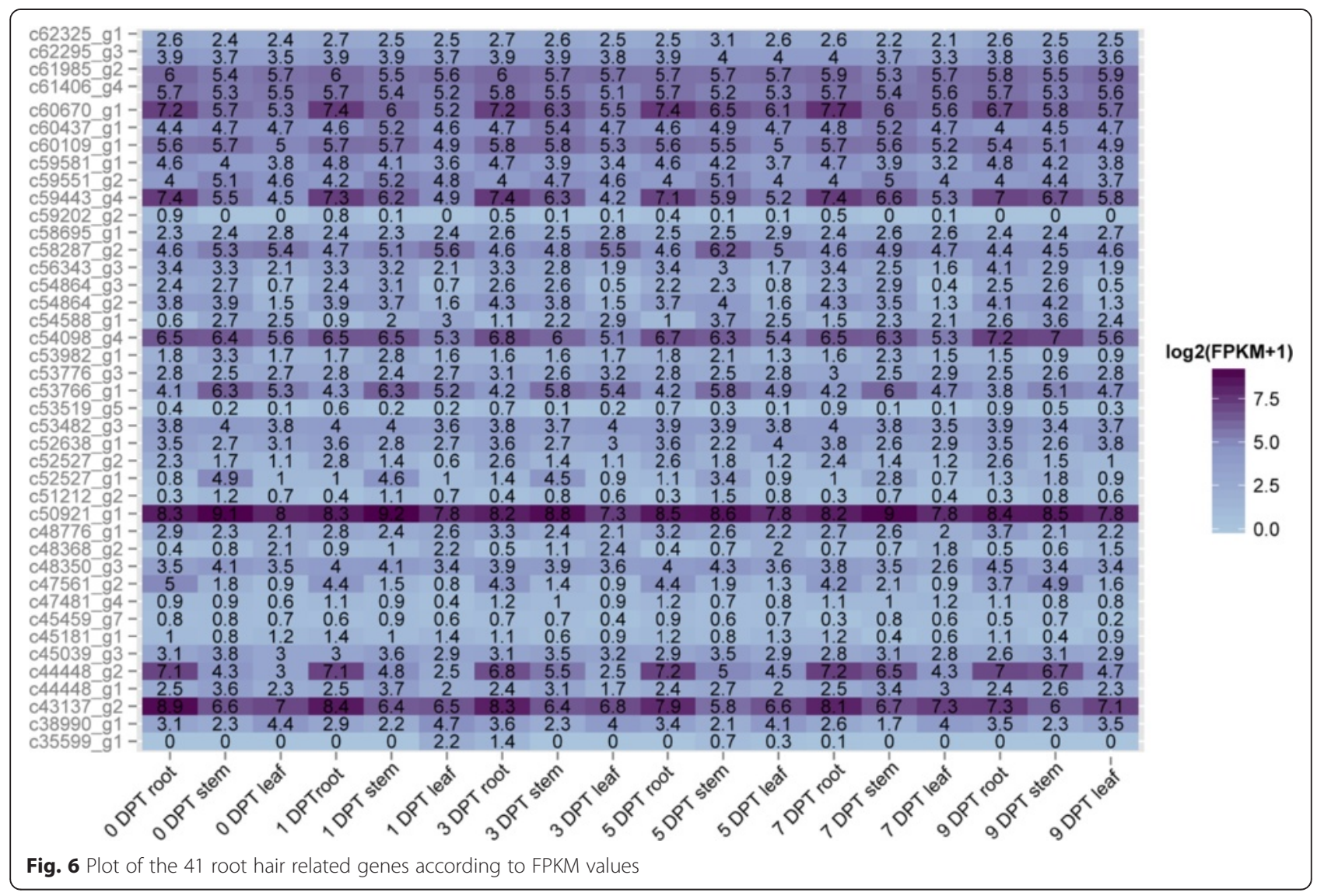

were up-regulated in roots $(P=5.8375 \mathrm{E}-3)$. Our results revealed that the expression of three AP2/ERF (c46110_g2, c46110_g3), four MYB (c34519_g1, c49349_g1, c55923_g2, c59091_g1), and nine WRKY genes (c46449_g1, c49987_g2, c49987_g1, c50394_g2, c51336_g1, c54471_g1, c55588_g2, c59948_g3, c46217_g1) were up-regulated in response to benzoic acid, suggesting that responses to benzoic acid might require the upregulated TFs in roots to regulate downstream response genes. The qRT-PCR validations and RNA-Seq profiles for these TFs could be found at Additional file 12.

\section{Alternative splicing of the transcriptome upon benzoic acid induction}

In eukaryotes, alternative splicing (AS) increases the diversity of the transcriptome and the proteome and takes part in biological process such as plant-virus interactions [54] and stress-related regulation [55]. However, the AS events upon benzoic acid treatment have never been reported. We used previous published method to identify AS events in a genome-wide way [25]. In brief, we used BLAT to compare Trinity assembled sequences with the following options: - tileSize $=18$-oneOff $=0$-minIdentity $=96$-out $=\operatorname{sim} 4-$ maxIntron $=10000$. Then the candidates were revealed according to the pair-wise comparisons to search for indels. Finally, Indels that were flanked by two sequentially matching sections were regarded as candidate AS regions. In total, 58,518 AS events in 12,950 AS genes were found upon benzoic acid induction. AS events from 17 randomly selected genes were validated by RT-PCR. Then the expected band sizes can be predicted according to the RNA-Seq results and were listed at Additional file 15. The real band sizes from RT-PCR that matched the expected band size from RNA-Seq were arrowed in Fig. 8. For genes c15452_g1 and c11325_g1, we only observed one clear expected band, however, the other weak bands might be caused by low abundance. The other 15 genes can be detected all expected bands and the obtained production sizes of RT-PCR were consistent with that of the RNA-Seq results. Splicing factors are extensively alternatively spliced. In this study, we found 113 splicing factors. Among these splicing factors, 61 splicing factors were regulated by AS. In total, the AS splicing factor percentage was $54 \%$, higher than the average AS ratio (18 \%). Among the contigs involved in the ginsenoside biosynthetic pathway, one AACT, one AS, 3 FPS, 1 GPS, 2 HMGR, 1 HMGS, 1 SE, 4 SS and 5 UGT contigs were regulated by AS. 


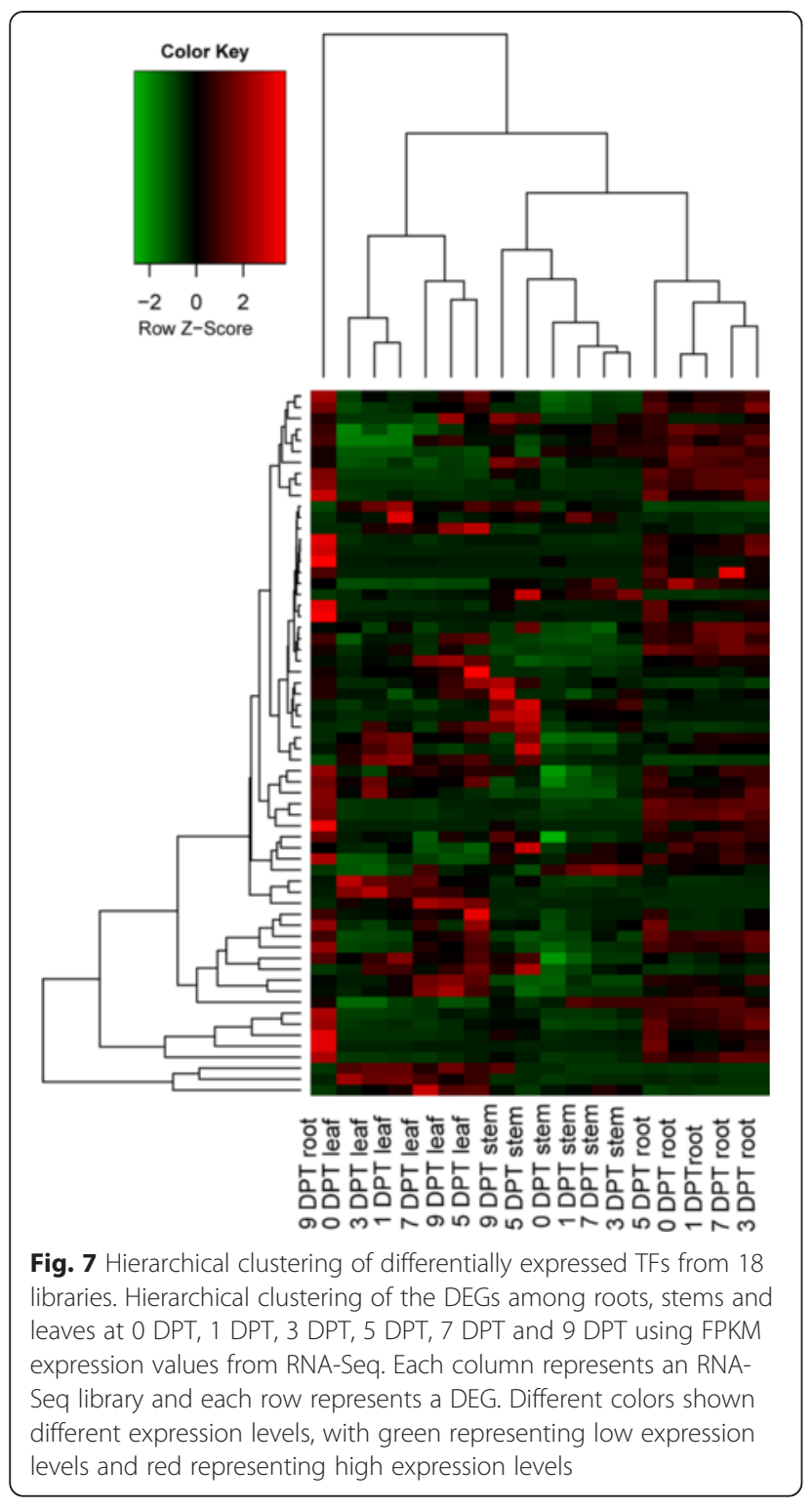

\section{Discussion}

Autotoxicity reduced both yield and quality of crops in a continuous cropping pattern [18]. P. ginseng, one of the most important traditional Chinese herbal medicines, exhibited a severe autotoxicity problem. In our previous study, secondary metabolites including benzoic acid were identified with strong autotoxic and growth-inhibiting activity on P. ginseng [33]. However, the molecular mechanism by which $P$. ginseng responds to benzoic acid remains unknown. In this study, we investigated the effect of benzoic acid on gene expression profiles using RNASeq technology and identified the differentially expressed genes that directly respond to autotoxins and the enriched GO terms that were involved in multiple biological processes. Moreover, we identified three up-regulated ROS-related genes. ROS can damage cell membranes and other components. The ROS-scavenging system involves three major enzymes: SOD, POD and CAT [46]. In this study, we found that SOD and POD contigs were up- regulated at later stages, which might enhance the antioxidation mechanisms at the later stage of benzoic acid induction. The physiological and biochemical indexes for proline and MDA also support this conclusion. Consistent with the gene expression profiles of SOD and POD, the proline and MDA content decreased at the later stage of benzoic acid treatment. Both free proline and MDA were restored to the lower level at a later stage (9 DPI), implying that $P$. ginseng partly became resistant to benzoic acid stress at the later stage.

We also found that two important contigs that coded for the enzymes $\mathrm{CHI}$ and $\mathrm{F} 3 \mathrm{H}$ in the flavonoid biosynthesis pathway were repressed at the later stage. Flavonoid production might be repressed at the later stage by the down-regulation of $\mathrm{CHI}$ and $\mathrm{F} 3 \mathrm{H}$ upon benzoic acid treatment. In this way, flavonoid could be presented at a relatively low concentration, and the exudation of flavonoid into soil could be reduced. The reduced concentrations of flavonoid in the soil can the release some of the adverse effects of autotoxin. In the future, the actual flavonoid concentrations upon benzoic acid treatment will be measured to obtain accurate information about the regulation of flavonoid biogenesis and transporters in the response to autotoxins.

Autotoxicity and pathogens are two major causes of continuous cropping $[6,56]$. Thus, several groups have investigated the interplay between autotoxins and pathogens in soil. Autotoxins can be degraded by pathogens

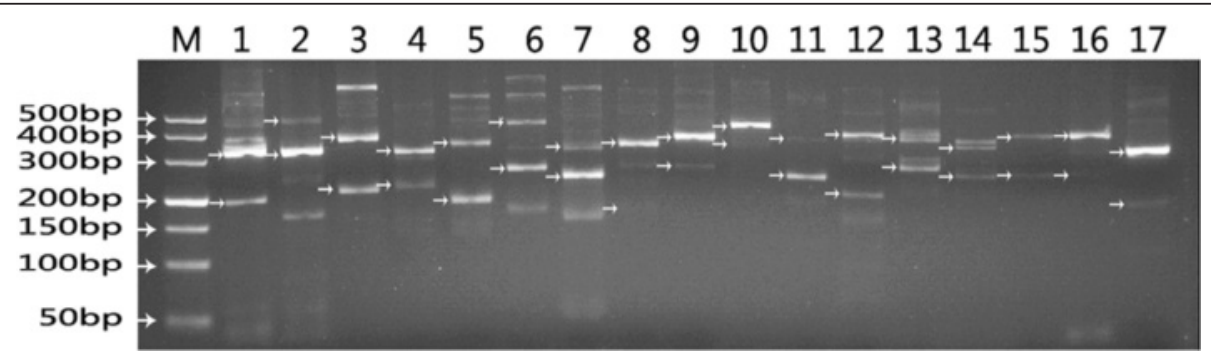

Fig. 8 Validation of randomly selected AS events by RT-PCR 
$[57,58]$ and repress the prevalence of soil pathogens [2]. However, another study showed that autotoxins exuded from the root of Ginseng saponins stimulated the growth of soil pathogens [59]. Baicalin released from Scutellaria baicalensis Georgi increases the activity of soil pathogens [14]. The suppression or enhancement effect of autotoxins on pathogens can be explained by the diversity of responses of plants to different autotoxins and pathogens. The accumulation of $C$. destructans, one of the most destructive pathogens, results in serious root rot disease and prevents the continuous cultivation of $P$. ginseng [60]. Autotoxins in the rhizosphere in $P$. ginseng affect and interact with pathogens in soils. However, a study on this topic has never been reported in P. ginseng. We still do not know the suppression or enhancement effects of autotoxins on the most destructive pathogens. In this study, we found that 'stress response genes' was enriched in differentially expressed genes after exposure to benzoic acid. These genes might also affect disease resistance upon Cylindrocarpon destructans infection. Understanding the interaction underlying autotoxins and pathogens is necessary to effectively control both $P$. ginseng root diseases and the adverse effects of autotoxicity.

Previous studies showed that the autotoxin potential of many plants is species-dependent [2]. The autotoxin was toxic to cucumber but not to fig leaf gourd plants [61]. In this study, we only uncovered the transcriptional response induced by benzoic acid. Thus, in the future it will be interesting to investigate different $P$. ginseng autotoxins to reveal the similarities and differences in the molecular mechanisms of autotoxininduced responses.

\section{Conclusions}

P. ginseng is a suitable plant for the study of autotoxicity because it is a highly valuable perennial herb and its biomass is affected by continuous cropping. Based on 18 RNA-Seq libraries from roots, stems, and leaves at different time points, we obtained transcriptome profiles and identified the DEGs upon benzoic acid stress. More importantly, changes in transcription factors upon benzoic acid induction were identified. Additionally, the study analyzed the reactive oxygen species genes and measured the physiological and biochemical indices of proline and MDA, which will help to reveal the molecular mechanisms of autotoxicity. Moreover, several differentially expressed root hair related genes that coded for key enzymes in the flavonoid biosynthesis pathway were identified, which might contribute to the dose-dependent inhibitory effect of benzoic acid on root hair development. Finally, the down-regulated contigs in the ginsenoside biosynthetic pathway were identified, and the repression of these contigs can reduce the biosynthesis of ginsenoside and partly release the adverse effects of autotoxins. This study provided a comprehensive characterization of the transcriptional response induced by autotoxins.

\section{Methods}

\section{Plant growth conditions and sample collection}

Roots of 3-year-old P. ginseng cv. Damaya were collected from the experimental field of the Institute of Medicinal Plant Development, Chinese Academy of Medical Sciences. Healthy P. ginseng roots were sterilized by $50 \%$ carbendazol wettable powder (diluted 800 times) for $10 \mathrm{~min}$ and then rinsed thrice in distilled water. The plants were transplanted into sterilized silica sand in plastic square pots $(40 \mathrm{~cm} \times 20 \mathrm{~cm} \times 15 \mathrm{~cm})$ with a density of 48 plants/pot and placed in a greenhouse under a $16 \mathrm{~h}$ light, $8 \mathrm{~h}$ dark period at $25{ }^{\circ} \mathrm{C}$ to control soil moisture, temperature, fertility, pathogens, and other unpredictable factors that may influence disease severity and pathogen growth. Hoagland nutrient solution was used to provide essential nutrients for the normal growth of the $P$. ginseng plants [62]. In this work, when the leaves of $P$. ginseng seedlings were completely spread, benzoic acid $\left(25 \mathrm{mg} \cdot \mathrm{L}^{-1}\right.$ ) was sprayed evenly on the sterilized silica sand with a volume of $200 \mathrm{~mL} /$ pot because benzoic acid with $25 \mathrm{mg} \cdot \mathrm{L}^{-1}$ significantly reduced root hair growth. The control was sprayed with Hoagland nutrient solution without benzoic acid.

\section{RNA extraction, library construction and sequencing}

The roots, stems and leaves of $P$. ginseng under benzoic acid stress were collected at 0 day, 1 day, 3 day, 5 day, 7 day and 9 day post treated (DPT). The samples were frozen in liquid nitrogen immediately after the surface was washed by distilled water. The tissues collected from the 0 day served as a control. In total, 18 samples were used to detect the global changes in gene expression. Total RNA was extracted using TRizol reagent according to the manufacturer's instructions and then digested with RNase-free DNase to eliminate the contamination of genomic DNA. Finally, an Agilent Technologies 2100 Bioanalyzer was used to measure and quantify the total RNA. The RNA samples with concentrations higher than $400 \mathrm{ng} \cdot \mathrm{ul}^{-1}$ and an RNA integrity number (RIN) > 8 were used for the RNA-Seq library construction and reverse transcription PCR (RT-PCR).

RNA-Seq libraries were constructed using Illumina's kit following the paired-end sample preparation kit protocol. In brief, the mRNA of each sample was enriched using the oligo (dT) magnetic beads. Then, purified RNA was cleaved into short fragments ( $330 \mathrm{nt})$ by adding fragmentation buffer prior to cDNA synthesis. Subsequently, the short fragments were ligated to sequencing adapters. 
Fragments with suitable sizes $(400 \sim 500 \mathrm{nt})$ were purified by agarose gel electrophoresis and then were selected to be templates for PCR amplification to produce the library and sequence via the Illumina HiSeq 2000 sequencing platform. All raw reads were pair-end sequenced and subjected to the following quality control to produce clean reads: (1) raw reads including adapter sequences and empty adapter were discarded; (2) reads including unknown $\mathrm{N}$ bases comprising more than $3 \%$ of the total length were filtered; (3) reads including low-quality bases that comprise more than $15 \%$ of total length were discarded. All following analyses were constructed on high quality reads.

\section{De novo transcriptome assembly and annotation}

De novo transcriptome assembly of the RNA-Seq reads was performed using Trinity (version: r20140413p1) software to obtain high-quality transcript sequences [34]. The Trinity in silico normalization utility was used to normalize the large RNA-Seq data sets. Then, Trinity was employed for de novo assembly using paired-end RNA-Seq reads with the default parameters. Transdecoder was used for open reading frame (ORF) identification using the default parameters [34]. The Kyoto Encyclopedia of Genes and Genomes (KEGG) database was used for obtaining KEGG metabolic pathway data.

\section{GO enrichment analysis}

To understand the biological functions of the differentially expression genes (DEGs), the assembled sequences were used for a homology search against the UniProt databases by ncbi-blast-2.2.27+ with an E-value of $10^{-6}$. The GO terms were assigned to assemble the sequences based on the UniProt databases (EMBL UniProt eggNOG/GO pathways databases). Then, all of the DEGs were mapped to the GO database and classified into three categories including "cellular component", "molecular function" and "biological process". The GO terms for DEGs were compared with the whole transcriptome background for GO enrichment analysis. GO enrichment analysis was performed on all DEGs using BINGO 3.0.2 [63] according to the custom GO annotation files from the transcriptome to identify the overrepresented GO terms in the DEGs. A $P$ value cutoff of 0.01 (hypergeometric test with Benjamini and Hochberg false discovery rate correction) was used to determine the enriched GO terms.

\section{Gene expression analysis (GEA) and the identification of DEGs}

GEA was performed in two sequential steps: (1) mapping all the clean reads to the assembled sequences using Bowtie2 2.2.3 [35] to calculate the read counts for each transcript and (2) measuring and normalizing the transcript abundances for each gene as the fragments per kilobase of exon per million fragments mapped (FPKM), which is analogous to single-read Reads Per Kilobase per Million mapped reads [64]. To more accurately calculate the expression level of the assembled genes, only both pairs of paired-end reads with unique location were retained to calculate expression values. We then calculated the $P$ value using the MA-plot-based method with random sampling model in the DEGseq $R$ package [36]. Then, genes with a fold change $>3$ and a $P<0.001$ were identified as DEGs. The hierarchical clustering of DEGs was performed using gplots in the $\mathrm{R}$ program environment using FPKM expression values in each RNA-Seq library.

\section{Validation of differential expression genes by qRT-PCR}

Total RNAs as described for RNA-Seq library construction were used for qRT-PCR. Reverse transcription was performed using $1 \mu \mathrm{g}$ total RNA for each sample and 200 U M-MLV Transcriptase (TaKaRa) in a $10 \mu \mathrm{l}$ volume. The reaction was conducted at $70{ }^{\circ} \mathrm{C}$ for $10 \mathrm{~min}, 42{ }^{\circ} \mathrm{C}$ for $60 \mathrm{~min}$ and $70{ }^{\circ} \mathrm{C}$ for $15 \mathrm{~min}$. The resulting cDNA was diluted to $800 \mu \mathrm{l}$ with sterile water. The qRT-PCR was conducted in triplicate reactions using the BIO-RAD CFX system (BIO-RAD). Gene-specific primers were designed by Primer3 (http://bioinfo.ut.ee/primer3/), and the primers for randomly selected 28 genes used in this study are listed in Additional file 16. The primers for validation of all the genes that described in the result part are listed in Additional file 17.

The 40S ribosomal protein S8 (c42687_g2) was used as the internal control for qRT-PCR because the expression level of this gene is relatively stable in samples of different tissues and different time points. The PCR was conducted in a $20 \mu \mathrm{l}$ volume containing $4 \mu \mathrm{l}$ diluted cDNA, $250 \mathrm{nM}$ forward primer, $250 \mathrm{nM}$ reverse primer, and $1 \times$ SYBR Premix Ex Taq II (TaKaRa) using the following conditions: $95^{\circ} \mathrm{C}$ for $3 \mathrm{~min}, 40$ cycles of $95{ }^{\circ} \mathrm{C}$ for $15 \mathrm{~s}, 59{ }^{\circ} \mathrm{C}$ for $15 \mathrm{~s}$ and $72{ }^{\circ} \mathrm{C}$ for $15 \mathrm{~s}$. Melting curve analyses were performed to verify the specificity using the Bio-Rad CFX Manage software. The relative expression levels were calculated using the $2^{-\Delta \Delta C T}$ method [65].

\section{Alternative splicing identification}

Because the $P$. ginseng genome is currently unavailable, we followed previous methods to identify alternative splicing (AS) events to obtain AS events based on assembled transcript sequences [25]. In brief, the Trinity assembled sequences were compared with each other by BLAT with the following options: - tileSize $=18$-oneOff $=0$ - minIdentity $=96$-out $=\operatorname{sim} 4-$ maxIntron $=10000$. Finally, the candidates were identified according to the 
pair-wise comparisons of assembled transcripts from the same locus to search for indels as candidate regions. Indels that were flanked by two sequentially matching sections from the pair comparison of assembled transcripts were regarded as candidate AS regions.

\section{Validation of AS events by RT-PCR}

Equal amounts of total RNA as described for RNA-Seq library construction were pooled and used for reverse transcription PCR (RT-PCR) to validate the AS events. Reverse transcription was carried out using $2 \mu \mathrm{g}$ of total RNA by 200 U M-MLV Reverse Transcriptase (TaKaRa) in a $20 \mu \mathrm{l}$ volume under the following the conditions: $65{ }^{\circ} \mathrm{C}$ for $5 \mathrm{~min} ; 25^{\circ} \mathrm{C}$ for $10 \mathrm{~min} ; 42^{\circ} \mathrm{C}$ for $60 \mathrm{~min}$ and $70{ }^{\circ} \mathrm{C}$ for $15 \mathrm{~min}$. The resulting cDNA was used for PCR amplification, which was performed under the following conditions: $95{ }^{\circ} \mathrm{C}$ for $3 \mathrm{~min}$; 45 cycles of $94{ }^{\circ} \mathrm{C}$ for $30 \mathrm{~s}$; $58{ }^{\circ} \mathrm{C}$ for $30 \mathrm{~s}$; and $72{ }^{\circ} \mathrm{C}$ for $15 \mathrm{~s}$. The PCR products were separated by electrophoresis with a $3 \%$ agarose gel. Primers used for the RT-PCR validation of AS events are listed in Additional file 15.

\section{Root morphology under benzoic acid treatment}

After ripening, the ginseng seeds were sterilized by $50 \%$ carbendazol wettable powder (diluted 800 times) for $10 \mathrm{~min}$ followed by rinsing three times in distilled water. Then, the seeds were cultivated in Petri dishes at $20{ }^{\circ} \mathrm{C}$ under dark conditions with five different concentrations of benzoic acid $\left(0,0.25,2.5,25,250 \mathrm{mg} \cdot \mathrm{L}^{-1}\right)$. Seven days later, the root morphology was observed at 10 times magnification by LeicaS8APO stereomicroscope via the Anymicro DSS YT-7 M microscopic digital camera system. Ten ginseng seedlings were observed for each concentrations of benzoic acid. In total, 50 ginseng seedlings were investigated in this study.

\section{Measurement of physiological and biochemical indexes}

The seedlings were cultivated in the same conditions as the sequencing materials. Then the seedlings were treated with four different concentrations of benzoic acid $\left(0,2.5,25,250 \mathrm{mg} \cdot \mathrm{L}^{-1}\right)$. The content of proline in the leaves was measured at $0,1,3,5,7$ and 9 DPT using the ninhydrin method [66]. The MDA content was measured using the thiobarbituric acid method [67]. The content of both proline and MDA were measured with three biological replicates.

\section{Additional files}

Additional file 1: Sequence length distribution of assembled transcripts. The $X$-axis indicates the length range of the transcript sequences. The $Y$-axis indicates the percentage of transcript sequences with a certain length. (PDF 81 kb)
Additional file 2: All the annotation information for the assembled transcripts. (XLS $26730 \mathrm{~kb}$ )

Additional file 3: Venn diagram showing annotated genes by SP, NR, NT, and KEGG. The number of annotated genes is listed in each diagram component. (PDF $112 \mathrm{~kb}$ )

Additional file 4: Plotting the distribution of log-transformed FPKM values across 18 libraries. The $X$-axis indicates the $\log 2$ of the FPKM value, and the $Y$-axis indicates the percentage of genes with a given FPKM value. (PDF $241 \mathrm{~kb}$ )

Additional file 5: The lists for all the differentially expressed genes. (XLS $1362 \mathrm{~kb}$ )

Additional file 6: The expression profiles based on RNA-Seq for $\mathbf{2 8}$ randomly chosen DEGs. (JPEG $1560 \mathrm{~kb}$ )

Additional file 7: The RNA-Seq profiles together with the qRT-PCR validations for DEGs across all the time points. (TIFF $1778 \mathrm{~kb}$ )

Additional file 8: The RNA-Seq profiles and qRT-PCR validations for the ROS-related genes. (TIFF $1356 \mathrm{~kb}$ )

Additional file 9: The RNA-Seq profiles and qRT-PCR validations for P5CS, P5CR and APX. (TIFF $747 \mathrm{~kb}$ )

Additional file 10: The RNA-Seq profiles and $\mathrm{qRT}$-PCR validations for root hair related genes. (TIFF $2900 \mathrm{~kb}$ )

Additional file 11: The RNA-Seq profiles and qRT-PCR validations for genes involved in flavonoid and ginsenoside biosynthetic pathways. (TIFF $1953 \mathrm{~kb}$ )

Additional file 12: The RNA-Seq profiles and qRT-PCR validations for TFs upon benzoic acid Treatment. (TIFF 2528 kb)

Additional file 13: The differentially expressed genes across all the time points. (XLS $66 \mathrm{~kb}$ )

Additional file 14: Benzoic acid inhibited the growth of root hairs. (JPEG $1687 \mathrm{~kb}$ )

Additional file 15: Primers of 17 genes for alternative splicing validation. (PDF $13 \mathrm{~kb}$ )

Additional file 16: Primers of 28 randomly chosen DEGs for validation. (PDF $506 \mathrm{~kb}$ )

Additional file 17: Primers of all the genes described in the result section for qRT-PCR validation. (PDF $91 \mathrm{~kb}$ )

\section{Abbreviations}

ANS: anthocyanidin synthase; AP2: APETALA 2 transcription factor; APX: ascorbate peroxidase; AS: alternative splicing; A3GT: anthocyanidin 3-O-glucosyltransferase; BLAST: basic local alignment search tool; CAT: Catalase; CHS: chalcone synthase; $\mathrm{CHI}$ : chalcone isomerase; CDNA: complementary DNA; DCF: dihydrodichlorofluorescein; DEG: differentially expressed gene; DPI: day post-inoculation; ERF: ethylene responsive factor; EMBL: European Molecular Biology Laboratory; FPKM: fragments per kilobase of exon per million fragment; $\mathrm{F} 3 \mathrm{H}$ : flavanone 3-hydroxylase; F3'5'H: Flavonoid 3' 5'-hydroxylase; F3'H: Flavonoid 3'hydroxylase; FA: Ferulic acid; GO: gene ontology; GEA: gene expression analysis; KEGG: kyoto encyclopedia of genes and genomes; MDA: malondialdehyde; MYB: transcription factor containing a Myb DNA binding domain; NAM: no apical meristem; NR: non-redundant protein database; NT: nucleotide database; ORF: open reading frame; POD: peroxidase; RNA-Seq: RNA sequencing; RT-PCR: reverse transcription-PCR; RPKM: Reads Per Kilobase per Million mapped reads; ROS: Reactive oxygen species; RIN: RNA integrity number;

SOD: superoxide dismutase; STEM: short time-series expression miner; WRKY: (WRKYGQK); TF: transcription factor.

Competing interests

We have no competing interests.

\section{Authors' contributions}

WD conceived and designed the experiments. BW, QL, YG, YL performed the experiments. BW, ZW and TS analyzed the data. YL, BW and WD wrote the paper. All the authors have read and approved the manuscript. 


\section{Acknowledgements}

This research was supported by the National Science Foundation of China (81072992 and 81373911). The funders had no role in study design, data collection and analysis, decision to publish, or preparation of the manuscript.

\section{Received: 17 October 2015 Accepted: 27 October 2015 Published online: 25 November 2015}

\section{References}

1. Bennett AJ, Bending GD, Chandler D, Hilton S, Mills P. Meeting the demand for crop production: the challenge of yield decline in crops grown in short rotations. Biol Rev Camb Philos Soc. 2012;87(1):52-71.

2. Huang LF, Song LX, Xia XJ, Mao WH, Shi K, Zhou YH, et al. Plant-soi feedbacks and soil sickness: from mechanisms to application in agriculture. J Chem Ecol. 2013;39(2):232-42.

3. Miller DA. Allelopathy in forage crop systems. Agron J. 1996;88:854-9.

4. Chou C-H, Lin H-J. Autointoxication mechanism of Oryza sativa L. Phytotoxic effects of decomposing rice residues in soil. J Chem Ecol. 1976;2(3):353-67.

5. Hao Z, Wang Q, Christie P, Li X. Allelopathic potential of watermelon tissues and root exudates. Sci Hortic. 2007;112(3):315-20.

6. Yang $M$, Zhang $X, X u Y$, Mei $X$, Jiang $B$, Liao J, et al. Autotoxic ginsenosides in the rhizosphere contribute to the replant failure of Panax notoginseng. PLoS One. 2015;10(2):e0118555

7. Chi WC, Fu SF, Huang TL, Chen YA, Chen CC, Huang HJ. Identification of transcriptome profiles and signaling pathways for the allelochemica juglone in rice roots. Plant Mol Biol. 2011;77(6):591-607.

8. Wu H, Pratley J, Lemerle D, An M, Li LD. Autotoxicity of wheat (Triticum aestivum L.) as determined by laboratory bioassays. Plant Soil. 2007;296(1-2):85-93.

9. Chon SU, Boo HO. Difference in allelopathic potential as influenced by root periderm colour of sweet potato (Ipomoea batatas). J Agron Crop Sci. 2005;191(1):75-80

10. Manici L, Ciavatta C, Kelderer M, Erschbaumer G. Replant problems in South Tyrol: role of fungal pathogens and microbial population in conventional and organic apple orchards. Plant Soil. 2003;256(2):315-24.

11. Chon S-U, Nelson C, Coutts J. Osmotic and autotoxic effects of leaf extracts on germination and seedling growth of alfalfa. Agron J. 2004:96(6):1673-9.

12. Gao W, Zhao Y, Wang Y, Chen S. A review of research on sustainable use of medicinal plants cropland in China. China J Chin Materia Medica. 2006;31(20):1665-9.

13. Sun $Y$, Lin S, Huang L, Zhang $X$, Guo L. Review: autotoxicity in medicinal plants and means to overcome. China J Chin Materia Medica. 2011;36(4):387-90

14. Zhang S, Jin Y, Zhu W, Tang J, Hu S, Zhou T, et al. Baicalin released from Scutellaria baicalensis induces autotoxicity and promotes soilborn pathogens. J Chem Ecol. 2010;36(3):329-38.

15. Ruan X, Li ZH, Wang Q, Pan CD, Jiang DA, Wang GG. Autotoxicity and allelopathy of 3,4-dihydroxyacetophenone isolated from Picea schrenkiana needles. Molecules. 2011;16(10):8874-93.

16. Hartung AC, Nair MG, Putnam AR. Isolation and characterization of phytotoxic compounds from asparagus (Asparagus officinalis L.) roots. J Chem Ecol. 1990;16(5):1707-18.

17. Singh HP, Batish DR, Kohli RK. Autotoxicity: Concept, organisms, and ecological significance. Crit Rev Plant Sci. 1999;18:757-72.

18. Zhou X, Wu F. p-Coumaric acid influenced cucumber rhizosphere soil microbial communities and the growth of Fusarium oxysporum f.sp. cucumerinum Owen. PLoS One. 2012;7(10):e48288.

19. Baerson SR, Sanchez-Moreiras A, Pedrol-Bonjoch N, Schulz M, Kagan IA, Agarwal AK, et al. Detoxification and transcriptome response in Arabidopsis seedlings exposed to the allelochemical benzoxazolin-2(3H)-one. J Biol Chem. 2005;280(23):21867-81.

20. Golisz A, Sugano M, Fujii Y. Microarray expression profiling of Arabidopsis thaliana $\mathrm{L}$. in response to allelochemicals identified in buckwheat. J Exp Bot. 2008;59(11):3099-109.

21. Golisz A, Sugano M, Hiradate S, Fujii Y. Microarray analysis of Arabidopsis plants in response to allelochemical L-DOPA. Planta. 2011:233(2):231-40.

22. Chi WC, Chen YA, Hsiung YC, Fu SF, Chou CH, Trinh NN, et al. Autotoxicity mechanism of Oryza sativa: transcriptome response in rice roots exposed to ferulic acid. BMC Genomics. 2013;14:351.

23. Wang Z, Gerstein M, Snyder M. RNA-Seq: a revolutionary tool for transcriptomics. Nat Rev Genet. 2009;10(1):57-63.
24. Filichkin SA, Priest HD, Givan SA, Shen R, Bryant DW, Fox SE, et al. Genomewide mapping of alternative splicing in Arabidopsis thaliana. Genome Res. 2010;20(1):45-58

25. Wu B, Suo F, Lei W, Gu L. Comprehensive Analysis of Alternative Splicing in Digitalis purpurea by Strand-Specific RNA-Seq. PLoS One. 2014;9(8):e106001.

26. Zhao S, Fung-Leung WP, Bittner A, Ngo K, Liu X. Comparison of RNA-Seq and microarray in transcriptome profiling of activated T cells. PLoS One. 2014;9(1):e78644.

27. Im GJ, Chang JW, Choi J, Chae SW, Ko EJ, Jung HH. Protective effect of Korean red ginseng extract on cisplatin ototoxicity in HEI-OC1 auditory cells. Phytother Res. 2010;24(4):614-21.

28. Li C, Zhu Y, Guo X, Sun C, Luo H, Song J, et al. Transcriptome analysis reveals ginsenosides biosynthetic genes, microRNAs and simple sequence repeats in Panax ginseng C. A. Meyer. BMC Genomics. 2013;14:245.

29. Luo H, Sun C, Sun Y, Wu Q, Li Y, Song J, et al. Analysis of the transcriptome of Panax notoginseng root uncovers putative triterpene saponinbiosynthetic genes and genetic markers. BMC Genomics. 2011;12 Suppl 5:S5

30. Mathiyalagan R, Subramaniyam S, Natarajan S, Kim YJ, Sun MS, Kim SY, et al. Insilico profiling of microRNAs in Korean ginseng (Panax ginseng Meyer). J Ginseng Res. 2013;37(2):227-47.

31. Sun C, Li Y, Wu Q, Luo H, Sun Y, Song J, et al. De novo sequencing and analysis of the American ginseng root transcriptome using a GS FLX Titanium platform to discover putative genes involved in ginsenoside biosynthesis. BMC Genomics. 2010;11:262.

32. He CN, Gao WW, Yang JX, Wu B, Zhang XS, Zhao YJ. Identification of autotoxic compounds from fibrous roots of Panax quinquefolium L. Plant Soil. 2009;318:63-72

33. Li Y, Huang XF, Ding WL. Autotoxicity of Panax ginseng rhizosphere and non-rhizosphere soil extracts on early seedlings growth and identification of chemicals. Allelopathy J. 2011;28(2):145-54.

34. Grabherr MG, Haas BJ, Yassour M, Levin JZ, Thompson DA, Amit I, et al. Full-length transcriptome assembly from RNA-Seq data without a reference genome. Nat Biotechnol. 2011;29(7):644-52.

35. Langmead B, Salzberg SL. Fast gapped-read alignment with Bowtie 2. Nat Methods. 2012:9(4):357-9.

36. Wang L, Feng Z, Wang X, Zhang X. DEGseq: an R package for identifying differentially expressed genes from RNA-seq data. Bioinformatics. 2010;26(1):136-8.

37. Zhang J, Pan Z, Moloney S, Sheppard A. RNA-Seq analysis implicates detoxification pathways in ovine mycotoxin resistance. PLoS One. 2014;9(6):e99975

38. Ernst J, Bar-Joseph Z. STEM: a tool for the analysis of short time series gene expression data. BMC Bioinformatics. 2006;7:191.

39. Janz D, Lautner S, Wildhagen H, Behnke K, Schnitzler JP, Rennenberg H, et al. Salt stress induces the formation of a novel type of 'pressure wood' in two Populus species. New Phytol. 2012;194(1):129-41.

40. Yu JQ, Matsui Y. Phytotoxic substances in root exudates of cucumber (Cucumis sativus L.). J Chem Ecol. 1994:20(1):21-31.

41. Holappa LD, Blum U. Effects of exogenously applied ferulic acid, a potential allelopathic compound, on leaf growth, water utilization, and endogenous abscisic acid levels of tomato, cucumber, and bean. J Chem Ecol. 1991;17(5):865-86.

42. Apel K, Hirt H. Reactive oxygen species: metabolism, oxidative stress, and signal transduction. Annu Rev Plant Biol. 2004;55:373-99.

43. Du F, Shi H, Zhang $X, X u X$. Responses of reactive oxygen scavenging enzymes, proline and malondialdehyde to water deficits among six secondary successional seral species in Loess Plateau. PLoS One. 2014;9(6):e98872.

44. Delauney AJ, Verma DPS. Proline biosynthesis and osmoregulation in plants. Plant J. 1993:4(2):215-23

45. Ashraf M, Foolad M. Roles of glycine betaine and proline in improving plant abiotic stress resistance. Environ Exp Bot. 2007:59(2):206-16.

46. Asada K. Ascorbate peroxidase - a hydrogen peroxide-scavenging enzyme in plants. Physiol Plantarum. 1992;85(2):235-241.

47. Weston LA, Mathesius U. Flavonoids: their structure, biosynthesis and role in the rhizosphere, including allelopathy. J Chem Ecol. 2013;39(2):283-97.

48. Kaltenbach M, Schroder G, Schmelzer E, Lutz V, Schroder J. Flavonoid hydroxylase from Catharanthus roseus: CDNA, heterologous expression enzyme properties and cell-type specific expression in plants. Plant J. 1999;19(2):183-93. 
49. Tanaka Y, Yonekura K, Fukuchi-Mizutani M, Fukui Y, Fujiwara H, Ashikari T, et al. Molecular and biochemical characterization of three anthocyanin synthetic enzymes from Gentiana triflora. Plant Cell Physiol. 1996;37(5):711-6.

50. Agarwal PK, Agarwal P, Reddy M, Sopory SK. Role of DREB transcription factors in abiotic and biotic stress tolerance in plants. Plant Cell Rep. 2006;25(12):1263-74

51. PARK MR, YUN KY, Mohanty B, Herath V, Xu F, Wijaya E, et al. Supra-optimal expression of the cold-regulated OsMyb4 transcription factor in transgenic rice changes the complexity of transcriptional network with major effects on stress tolerance and panicle development. Plant Cell Environ. 2010;33(12):2209-30.

52. Shin R, Park JM, An J-M, Paek K-H. Ectopic expression of Tsi1 in transgenic hot pepper plants enhances host resistance to viral, bacterial, and oomycete pathogens. Mol Plant-Microbe Interact. 2002;15(10):983-9.

53. Wang H, Hao J, Chen $X$, Hao Z, Wang X, Lou Y, et al. Overexpression of rice WRKY89 enhances ultraviolet $B$ tolerance and disease resistance in rice plants. Plant Mol Biol. 2007;65(6):799-815.

54. Mandadi KK, Scholthof K-BG. Genome-wide analysis of alternative splicing landscapes modulated during plant-virus interactions in Brachypodium distachyon. Plant Cell. 2015;27(1):71-85.

55. Kazan K. Alternative splicing and proteome diversity in plants: the tip of the iceberg has just emerged. Trends Plant Sci. 2003;8(10):468-71.

56. Lebreton L, Lucas P, Dugas F, Guillerm AY, Schoeny A, Sarniguet A. Changes in population structure of the soilborne fungus Gaeumannomyces graminis var. tritici during continuous wheat cropping. Environ Microbiol. 2004;6(11):1174-85

57. Weidenhamer JD, Li M, Allman J, Bergosh RG, Posner M. Evidence does not support a role for gallic acid in Phragmites australis invasion success. J Chem Ecol. 2013;39(2):323-32

58. Blum U, Staman KL, Flint LJ, Shafer SR. Induction and/or selection of phenolic acid-utilizing bulk-soil and rhizosphere bacteria and their influence on phenolic acid phytotoxicity. J Chem Ecol. 2000;26(9):2059-78.

59. Nicol RW, Yousef $L$, Traquair JA, Bernards MA. Ginsenosides stimulate the growth of soilborne pathogens of American ginseng. Phytochemistry. 2003;64(1):257-64

60. Rahman M, Punja ZK. Factors Influencing Development of Root Rot on Ginseng Caused by Cylindrocarpon destructans. Phytopathology. 2005;95(12):1381-90.

61. Ding J, Sun Y, Xiao CL, Shi K, Zhou YH, Yu JQ. Physiological basis of different allelopathic reactions of cucumber and figleaf gourd plants to cinnamic acid. J Exp Bot. 2007:58(13):3765-73.

62. Taffouo VD, Ngwene B, Akoa A, Franken P. Influence of phosphorus application and arbuscular mycorrhizal inoculation on growth, foliar nitrogen mobilization, and phosphorus partitioning in cowpea plants. Mycorrhiza. 2014;24(5):361-8.

63. Maere S, Heymans K, Kuiper M. BiNGO: a Cytoscape plugin to assess overrepresentation of gene ontology categories in biological networks. Bioinformatics. 2005;21(16):3448-9.

64. Mortazavi A, Williams BA, McCue K, Schaeffer L, Wold B. Mapping and quantifying mammalian transcriptomes by RNA-Seq. Nat Methods. 2008;5(7):621-8.

65. Livak KJ, Schmittgen TD. Analysis of relative gene expression data using real-time quantitative PCR and the $2^{-\Delta \Delta C T}$ Method. Methods. 2001;25(4):402-8

66. Bates $L$, Waldren $R$, Teare I. Rapid determination of free proline for water-stress studies. Plant Soil. 1973:39(1):205-7.

67. Uchiyama M, Mihara M. Determination of malonaldehyde precursor in tissues by thiobarbituric acid test. Anal Biochem. 1978;86(1):271-8.

\section{Submit your next manuscript to BioMed Centra and take full advantage of:}

- Convenient online submission

- Thorough peer review

- No space constraints or color figure charges

- Immediate publication on acceptance

- Inclusion in PubMed, CAS, Scopus and Google Scholar

- Research which is freely available for redistribution

Submit your manuscript at

www.biomedcentral.com/submit
() BioMed Central 